\title{
Ostomy Site
}

National Cancer Institute

\section{Source}

National Cancer Institute. Ostomy Site. NCI Thesaurus. Code C122638.

The anatomic location of a surgically created ostomy. 